\title{
Comparison and real-time monitoring of high-order harmonic generation in different sources
}

\author{
J.-P. Brichta, ${ }^{1}$ M. C. H. Wong, ${ }^{1}$ J. B. Bertrand, ${ }^{1,2}$ H.-C. Bandulet, ${ }^{3}$ D. M. Rayner, ${ }^{2}$ and V. R. Bhardwaj ${ }^{1, *}$ \\ ${ }^{1}$ Department of Physics, University of Ottawa, 150 Louis-Pasteur, Ottawa, Ontario, Canada K1N 6N5 \\ ${ }^{2}$ National Research Council of Canada, 100 Sussex Drive, Ottawa, Ontario, Canada K1A OR6 \\ ${ }^{3}$ Institut National de la Recherche Scientifique-Centre Énergie, Matériaux et Télécommunications, 1650 boulevard Lionel-Boulet, \\ Varennes, Québec, Canada J3X 1S2
}

(Received 8 October 2008; published 11 March 2009)

\begin{abstract}
We compare the generation of coherent extreme ultraviolet (xuv) photons in argon from three different high-order harmonic sources: a semi-infinite gas cell, a finite gas cell, and a pulsed valve. We demonstrate photoionization of the background gas by the coherent xuv photons as an alternative to xuv spectroscopy for real-time and in-line monitoring of the high-order harmonics. Using this technique for measuring photocurrent, we show that the gas cells produce 50-100-fold brighter harmonics than the pulsed valve, with an estimated conversion efficiency of $10^{-4}$. The spectral distribution of the harmonics produced in the gas cells peaks at the 27 th harmonic compared to the 19th harmonic in the pulsed valve. We attribute this difference to the interplay between phase matching and absorption.
\end{abstract}

DOI: 10.1103/PhysRevA.79.033404

PACS number(s): $32.80 . R m, 42.65 . K y$

\section{INTRODUCTION}

Advances in ultrafast laser technology have enabled the development of table top sources of xuv and soft x-ray light through the process of high harmonic generation (HHG) $[1,2]$. High energy photons are produced in a nonlinear medium when an electron, removed from an atom or a molecule by the laser field, is driven back to the ion and undergoes recombination $[3,4]$. HHG opens new frontiers in science by extending nonlinear optics into the xuv region [5] and pushing ultrafast science to the attosecond domain [6], thus enabling: xuv spectroscopy, imaging of molecular orbitals [7], and electron motion. However, progress in attosecond science is presently hindered by the lack of high brightness harmonic sources. Research has been ongoing to improve HHG efficiency.

The efficiency of the HHG process depends on: (a) a high density of atoms leading to coherent buildup of light as it propagates through the nonlinear medium, (b) the length of the nonlinear medium, (c) absorption of harmonics by the nonlinear medium, and (d) phase velocity matching between the fundamental and the harmonic fields. In addition to medium dispersion, the presence of free electrons can lead to a phase velocity mismatch, which limits coherent buildup to short interaction lengths. The HHG efficiency is optimized by a delicate balance of these competing processes.

HHG from noble atoms in gas phase is the most widely used technique. However, laser ablation of solids provides an alternative, especially at lower harmonics [8]. Gas jets produced by a pulsed valve (PV) are a common source of high harmonics with typical medium lengths of 1-2 $\mathrm{mm}$ and a maximum on-axis pressure of 10 Torr. Conversion efficiencies of about $10^{-5}$ in the $30 \mathrm{~nm}$ [9] and $10^{-7}$ in the $13 \mathrm{~nm}$ $[10,11]$ region were obtained using high-power IR laser pulses and a loose focusing geometry. The efficiency of HHG in gas jets is determined solely by phase matching and

*ravi.bhardwaj@uottawa.ca is dominated by Gouy phase shift and medium dispersion. It is optimized by controlling the relative positions of the laser focus and the gas jet to balance both the Gouy phase shift and dipole phase shift [12]. A technical limitation of such a source is the repetition rate of the pulsed valve, which is typically much less than that of the laser.

In contrast, static gas cells permit the use of higher atomic densities and longer medium lengths, leading to higher conversion efficiencies due to their quadratic dependence. In addition to phase matching, absorption of harmonics by the medium starts to play an important role as the medium length gets longer [13]. For interaction lengths on the order of the confocal parameter of the laser, as in the case of finite gas cells (FGC), conversion efficiencies of $10^{-6}$ have been reported [14]. In semi-infinite gas cells (SIGC) [15], where the interaction length is several times the confocal parameter, pressures in excess of 100 Torr were used for efficient generation of high harmonics.

High-order harmonics can be generated in hollow capillary tubes [16] with conversion efficiencies of greater that $10^{-6}$, producing more that $0.2 \mathrm{~nJ}$ per harmonic order from a modest $150 \mu \mathrm{J}$ laser pulse. The efficiency of high harmonics generated in the above sources can be further enhanced by employing techniques such as adaptive pulse shaping, both in the frequency [17] and spatial domains [18], and by utilizing orthogonally polarized two-color (fundamental and frequency-doubled) driving lasers [19]. Despite the numerous studies on HHG efficiency in different types of sources, there is a lack of direct comparison between them.

In this paper, we use argon as the medium to compare HHG in a gas jet, a finite gas cell, and a semi-infinite gas cell. We show that the gas cells produce 50-100 times more intense harmonics relative to the gas jet. This direct relative comparison among the different sources was made possible by introducing an alternate technique of monitoring the harmonics by measuring the photocurrent produced ionization of the background gas by the high energy photons. The standard technique involves using an x-ray spectrometer. While the spectrometer provides spectral information, the efficiency of the microchannel plate detector varies with wavelength 

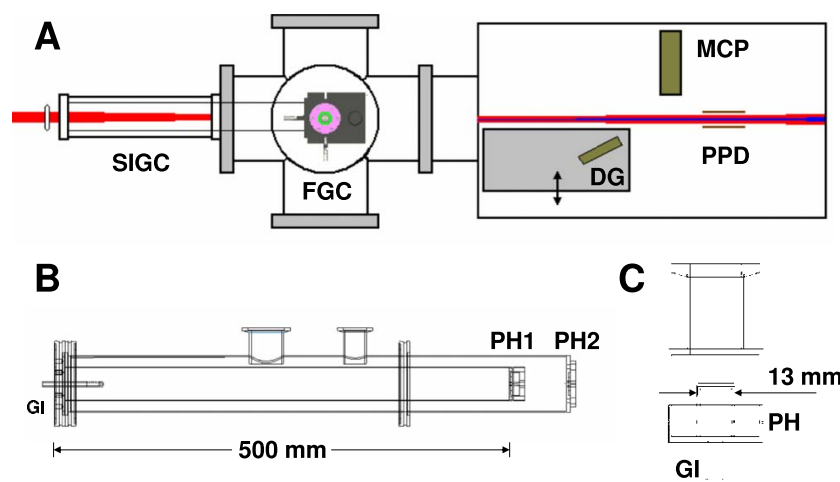

FIG. 1. (Color online) (a) Experimental setup for generation of xuv photons. SIGC, semi-infinite gas cell; FGC, finite gas cell; DG, dispersive grating. The grating sits on a motorized stage and must be removed from the beam to measure photocurrent with the parallel plate detector; MCP, microchannel plates with phosphor screen; PPD, parallel-plate detector. (b) Semi-infinite gas cell. GI, gas input; PH1, pinhole $(100-250 \mu \mathrm{m})$; $\mathrm{PH} 2,1 \mathrm{~cm}$ pinhole. (c) Finite gas cell. GI, gas input; $\mathrm{PH}, 4 \times 600 \mu \mathrm{m}$ pinholes.

and absolute calibration is difficult. Often used in conjunction with a spectrometer, an $\mathrm{x}$-ray photodiode measures short wavelength photons but must be calibrated with known sources such as a synchrotron. Unlike the standard methods, our technique can monitor high harmonics in real time and in-line, without intervention in or depletion of the x-ray beam. This is important for many studies in the attosecond domain.

\section{EXPERIMENT}

The schematic of the experimental setup for HHG is shown in Fig. 1. Linearly polarized laser pulses from a Ti:sapphire regenerative amplifier $(0.8 \mathrm{~mJ}, 35 \mathrm{fs}, 800 \mathrm{~nm}$, $100 \mathrm{~Hz}$ ) are focused through a $1 \mathrm{~mm}$ thick fused silica window onto the gas target with an achromatic lens of focal length $75 \mathrm{~cm}$. The target is either a gas jet produced by a pulsed valve or two different types of gas cells. In all cases, the harmonics are produced at the same location in the source chamber. The source and detector chambers are differentially pumped through a $3 \mathrm{~mm}$ diameter tube that is 5 $\mathrm{cm}$ in length. The different harmonic orders are detected using a grazing-incidence concave grating (1200 lines $\mathrm{mm}^{-1}$ ) which disperses and focuses them onto a microchannel plate $(15 \mathrm{~mm} \times 75 \mathrm{~mm})$ coupled to a phosphor screen. The screen is imaged with a charge coupled device (CCD) camera. In the typical operating configuration, harmonic orders 11-49 are detected. The harmonics are optimized by slightly moving the laser focus with respect to the target and by adjusting the laser chirp.

When the grating, positioned on a translation stage, is removed from the laser path, the harmonic beam passes through a copper parallel plate detector. The parallel plate detector and microchannel plates are equidistant from the location of high-order harmonic generation. The active area of the detector plates is approximately $10 \mathrm{~mm} \times 75 \mathrm{~mm}$ with an $8 \mathrm{~mm}$ separation. Ions of the background gas in the detection chamber $\left(P \sim 5 \times 10^{-7}\right.$ Torr $)$ created between the parallel plates by the high harmonic beam are detected by applying a bias of $+100 \mathrm{~V}$ on one of the plates and measuring the photocurrent on the other plate with a picoammeter. A larger bias voltage does not improve the signal collection efficiency. Since a major constituent of the background gas is nitrogen, which has an ionization potential of $15.58 \mathrm{eV}$, harmonics of the 11th order or higher are required for single photon ionization. As a result, the photocurrent scales linearly with photon flux. Since harmonics lower than the 11th order are also produced, our photocurrent measurement underestimates the total high harmonic flux. A small amount of argon is also present in the background.

Both of our gas cells employ a differential pumping geometry to reduce the rate of gas flow into the source and detection chambers. The SIGC consists of a cylindrical inner tube (500 $\mathrm{mm}$ long, $\phi 36 \mathrm{~mm}$ ) surrounded by a cylindrical outer tube (575 mm long, $\phi 60 \mathrm{~mm}$ ). Up to 200 Torr of gas pressure is delivered into the inner tube by a $\phi 6 \mathrm{~mm}$ stainless steel tube. The gas pressure is monitored by a Baratron gauge. The inner and outer tubes are separated by a $150 \mu \mathrm{m}$ diamond pinhole (Fort Wayne Wire Die, Inc.). The outer tube has a $1 \mathrm{~mm}$ pinhole and is pumped by a roughing pump $\left(500 \mathrm{~L} \mathrm{~min}^{-1}\right)$. The laser is focused to a spot directly before the diamond pinhole, about $50 \mathrm{~mm}$ from the center of the source chamber.

The FGC consists of a small cylindrical inner cell $(16 \mathrm{~mm}$ tall, $\phi 11 \mathrm{~mm})$ and larger cylindrical outer cell $(28 \mathrm{~mm}$ tall, $\phi 30 \mathrm{~mm})$. Gas is delivered to the inner cell through a $6 \mathrm{~mm}$ Teflon tube and pressure is monitored with a Baratron gauge. The inner cell has two inline pinholes $(\phi 600 \mu \mathrm{m})$ that lead to the outer cell. The outer cell also has two inline pinholes $(\phi 600 \mu \mathrm{m})$ which lead to the source chamber. The outer cell is pumped by a roughing pump $\left(500 \mathrm{~L} \mathrm{~min}^{-1}\right)$ similar to the SIGC. The FGC has an effective interaction length of $\sim 15 \mathrm{~mm}$ and is mounted on an $X Y Z$ manipulator to permit precision alignment with respect to the laser axis.

The PV has a $500 \mu \mathrm{m}$ aperture with a $200 \mu$ s opening time and operates at $10 \mathrm{~Hz}$ to avoid overpressuring the source chamber. The pulsed valve is mounted on an $X Y Z$ manipulator and is positioned so that the interaction length is $\sim 1 \mathrm{~mm}$. Stagnation pressures of up to $5 \mathrm{~atm}$ were used. We estimated the on-axis pressure in the gas jet based on dynamical arguments [20]. Specifically, for a stagnation pressure of $P_{0}$, the pressure in the gas jet, $P$, is given by

$$
\frac{P_{0}}{P}=\left(1+\frac{\gamma-1}{2} M^{2}\right)^{\gamma /(\gamma-1)}
$$

where $\gamma=5 / 3$ for a monatomic gas, $M=A(x / D)^{\gamma-1}$ is the Mach number, $A$ is a constant that depends on $\gamma$ and is 3.26 for a monatomic gas [21], $x$ is the distance from the nozzle, and $D$ is the nozzle diameter. With the laser positioned at a typical downstream distance of $\sim 1 \mathrm{~mm}$, a stagnation pressure of 2300 Torr produces an on-axis pressure of 7 Torr. The atomic density in a gas jet follows a Lorentzian distribution, and over the length of the laser-jet interaction region the pressure should be at least half of the on-axis pressure [22] or approximately 3.5 Torr. A fast ionization gauge from Beam-Dynamics, located below the pulsed valve, is used to 


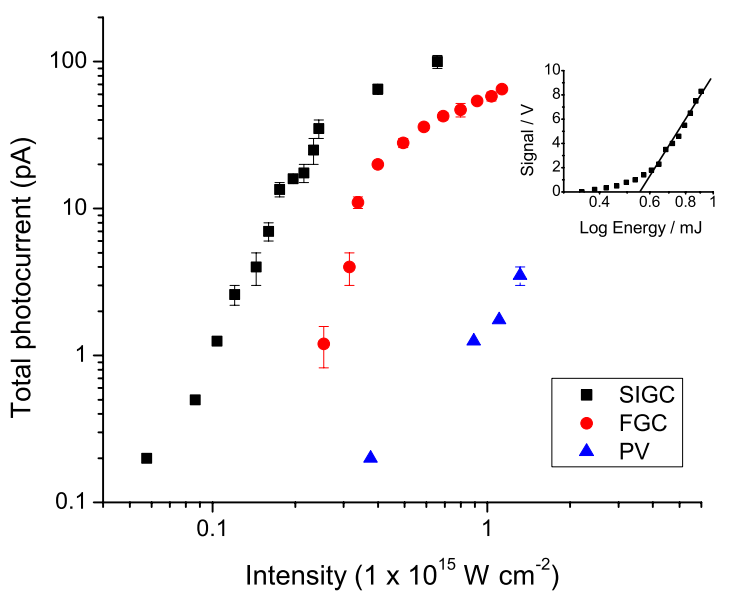

FIG. 2. (Color online) Total photocurrent as a function of laser intensity for different gas cell geometries. The photocurrent was recorded under optimum pressure conditions which were 43 Torr for the semi-infinite gas cell (black squares), 7 Torr for the finite gas cell (red circles), and 3.5 Torr for the pulsed valve (blue triangles). The inset graph shows the intensity calibration performed with the pulsed valve and a fast ionization gauge (see text). It indicates a saturation energy of $560 \mu \mathrm{J}$, which for argon corresponds to the saturation intensity of $2.37 \times 10^{14} \mathrm{~W} \mathrm{~cm}^{-2}$.

monitor the gas density and the position of the laser focus with respect to the gas jet by measuring the relative ion signal produced by the laser.

\section{RESULTS AND DISCUSSION}

For the three HHG sources that were investigated, the total photocurrent measured by the parallel plate detector as a function of the laser intensity is shown in Fig. 2. For each source an experimentally determined optimum gas pressure, $P$, was used. The photocurrent, vis-à-vis the harmonic signal from the SIGC ( $P=25$ Torr), grows by nearly 3 orders of magnitude over an intensity range of $5 \times 10^{13} \mathrm{~W} \mathrm{~cm}^{-2}-2 \times 10^{14} \mathrm{~W} \mathrm{~cm}^{-2}$, beyond which the signal starts to saturate. A similar trend is observed for the FGC photocurrent $(P=7.0$ Torr) in the intensity range of $2 \times 10^{14} \mathrm{~W} \mathrm{~cm}^{-2}-4 \times 10^{14} \mathrm{~W} \mathrm{~cm}^{-2}$. However, the harmonic signal is lower by a factor of 2-20 compared to the SIGC. In contrast, a relatively large intensity of $\sim 3 \times 10^{14} \mathrm{~W} \mathrm{~cm}^{-2}$ is required to record any photocurrent when using the PV (stagnation pressure of $2 \mathrm{~atm}$ ) and the photocurrent is at least 2 orders of magnitude smaller than the SIGC. Unlike in the gas cells, no saturation of the harmonic signal is observed until the maximum intensity is reached. In all sources, the lowest measurable photocurrent is $0.2 \mathrm{pA}$, which is limited by our ability to distinguish the signal from the background current of $<0.05 \mathrm{pA}$. The error bars on the vertical axis represent fluctuations in the harmonic signal due to shot-to-shot variations in pulse energy.

The observed saturation of the photocurrent (harmonic signal) in the gas cells is the result of a combination of neutral atom depletion and the free-electron contribution to phase mismatch. The laser intensity is accurately determined by measuring the saturation intensity of argon atoms as

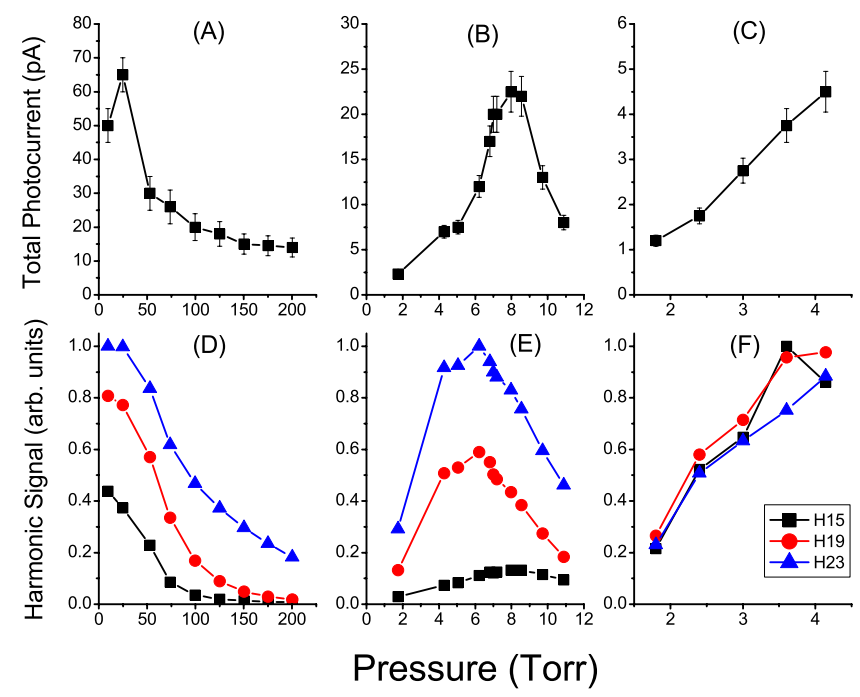

FIG. 3. (Color online) Pressure dependence of total photocurrent (top panel) and individual harmonics (bottom panel) measured using the x-ray spectrometer for $[(a)$ and (d)] the semi-infinite gas cell, $[(\mathrm{b})$ and (e)] the finite gas cell, and $[(\mathrm{c})$ and (f)] the pulsed valve. The laser intensity was $3 \times 10^{14} \mathrm{~W} \mathrm{~cm}^{-2}$ for the gas cells and $6 \times 10^{14} \mathrm{~W} \mathrm{~cm}^{-2}$ for the pulsed valve. Error bars are a result of shot-to-shot variation in the recorded photocurrent. In the bottom panel, the harmonics shown are the 15th (black squares), 19th (red circles), and 23rd (blue triangles).

shown in the inset of Fig. 2. We used the fast ionization gauge below the pulsed valve to record the ions produced by the laser as a function of pulse energy. When plotted in a semi-log plot the intercept on the energy axis gives the saturation intensity [23]. The saturation intensity of argon is $2.4 \times 10^{14} \mathrm{~W} \mathrm{~cm}^{-2}$.

Optimum gas pressures used in Fig. 2 are obtained by studying the pressure dependence of the total photocurrent (top panel in Fig. 3) and the intensity of individual harmonics with the xuv spectrometer (bottom panel in Fig. 3). For the latter, the signal strengths for $\mathrm{H} 13-\mathrm{H} 23$ were measured by integrating across the MCP image in those regions where the harmonics appeared. All three HHG sources have very different pressure dependencies.

In the SIGC, the harmonic signal reaches a maximum near 25 Torr and rapidly decreases due to stronger absorption of the harmonics at higher pressures. At 25 Torr, the typical absorption length is about $0.5 \mathrm{~mm}$ for the 15 th and 19 th harmonics whereas it is twice as long for the 23rd harmonic, leading to a stronger signal. On the other hand, the signal strength from the FGC rises at first due to increasing number density, maximizes at a pressure of 7 Torr, and then decreases as the absorption dominates over the $15 \mathrm{~mm}$ effective interaction length. Finally, the PV never reaches a critical point at which the harmonic signal is limited by absorption. At a pressure of 3.5 Torr, the absorption length would be approximately $4 \mathrm{~mm}$, which is much longer than the interaction length. As a result, the signal strength rises linearly with stagnation pressure. With their larger number densities, gas cells are essential for efficient harmonic generation.

We now show that the photocurrent measured by the parallel plate detector is due to photoionization of the back- 


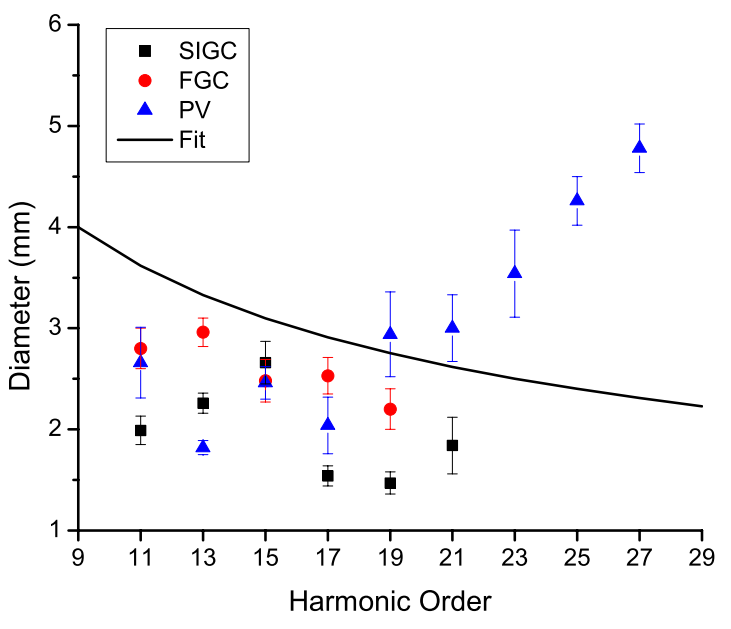

FIG. 4. (Color online) Diameter of harmonic beam at parallel plate detector measured for the semi-infinite gas cell (black squares), finite gas cell (red circles), and pulsed valve (blue triangles). The fit line plots the relationship $D_{0} / \sqrt{q}$, where $D_{0}$ is 12 $\mathrm{mm}$ in this case.

ground gas by the high order harmonics. First, by removing argon from the gas cells and turning off the gas jet, the measured photocurrent dropped to the background value. Second, by increasing the background gas density in the detection chamber, the photocurrent signal increased proportionately, as expected in single photon ionization. Third, by measuring the diameter of the harmonic beam at the detector, we ensured that the observed signal was due to ions produced and not due to the photoelectric effect. Since copper has a work function of $4.7 \mathrm{eV}$, harmonics higher than the third order could produce photoelectrons if they are incident on the detector. To measure the diameter of the harmonic beam we used the spectral images obtained by the xuv spectrometer. Since the harmonics are focused by the grating along one axis, their spatial extent along the other axis provides beam divergence.

The diameter of the harmonic beam for different orders at the parallel plate detector is shown for all three sources in Fig. 4. The recorded beam diameter is substantially less than the $8 \mathrm{~mm}$ separation between the parallel plates, which confirms that the photoelectric effect does not contribute to the measured photocurrent. For both gas cells, the beam diameter tends to decrease with increasing harmonic order. This is expected from the simple expression of the dipole moment at the $q$ th harmonic frequency, which according to lowest order perturbation theory, varies as the $q$ th power of the incident field. The solid line in Fig. 4 is the fit to $D_{0} / \sqrt{q}$, where $D_{0}$ is the diameter of the fundamental, measured to be $12 \mathrm{~mm}$. However, for the PV, the beam diameter increases slightly with the harmonic order, as observed in previous experiments [24]. The spatial characteristics of the harmonics depend on (a) the position of the laser focus with respect to the gas jet, and (b) on the spatial profile of the driving field. Divergence of harmonics was found to change by varying the aperture size near the input window. Unlike the earlier experiments with semi-infinite gas cells [15], we did not observe any aperture effect on the beam divergence or HHG intensity.
From the total photocurrent measured by the parallel plate detector we can estimate the HHG conversion efficiency. This estimate will be greatly influenced by any errors in our assumptions of the number of ions, the molecular density, the size of the harmonic beam, the interaction volume, and the ionization cross sections. Assuming the background molecules in the detection chamber are mostly nitrogen and that each molecule is singly ionized by the high harmonic beam, $100 \mathrm{pA}$ of measured photocurrent corresponds to $6.24 \times 10^{8}$ ions $\mathrm{s}^{-1}$. Harmonics lower than the 11th order cannot contribute to the photocurrent signal, assuming the probability for multiphoton ionization to be low. The number of ions $\mathrm{s}^{-1} \mathrm{~cm}^{-3}, r_{i}$, produced by the harmonic beam can be expressed as $r_{i}=\rho \sigma J_{\mathrm{HHG}}$, where $\rho$ is the molecular density (molecules $\mathrm{cm}^{-3}$ ) in the interaction region, $\sigma$ is the photoionization cross section measured in $\mathrm{cm}^{2}$, and $J_{\mathrm{HHG}}$ is the harmonic photon flux measured in photons $\mathrm{s}^{-1} \mathrm{~cm}^{-2}$. Assuming that only ions produced in the detector volume will contribute to the measured signal, $r_{i}=N_{i} / V=1.04 \times 10^{8}$ ions $\mathrm{s}^{-1} \mathrm{~cm}^{-3}$, where $N_{i}=6.24 \times 10^{8}$ ions s$^{-1}$ (as above) and $V=6 \mathrm{~cm}^{3}$. With a constant pressure of $1 \times 10^{-6}$ Torr, $\rho \sim 2.45 \times 10^{10} \mathrm{~cm}^{-3}$.

The photoionization cross section of $\mathrm{N}_{2}$ for each harmonic energy is taken from [25] and ranges from $\sigma$ $\simeq 8-25 \times 10^{-18} \mathrm{~cm}^{2}$ over the spectral range involving 11-49 harmonics. $J_{\mathrm{HHG}}$ is then calculated as a function of harmonic order and has a typical value of $\sim 3 \times 10^{14}$ photons $\mathrm{s}^{-1} \mathrm{~cm}^{-2}$. The power out is then determined by $P_{\mathrm{out}}=J_{\mathrm{HHG}} A E$, where $A$ is the area of the $q$ th harmonic at the detector, approximated by $A=\pi(1.2 / 2 \sqrt{q})^{2}$, and $E$ is the single-photon energy of the $q$ th harmonic multiplied by the relative abundance of that harmonic, as determined by the spectral image obtained with the spectrometer. Given an input power of $80 \mathrm{~mW}$, we estimate single order conversion efficiencies as high as $10^{-4}$ for the 27 th harmonic produced in a semi-infinite gas cell.

The three sources used in the experiment produced different harmonic spectra as shown in Fig. 5. The SIGC spectrum shows a relatively narrow distribution, with more than half of the signal coming from the 23rd to 31 st harmonic orders. The most intense harmonic is the 27 th. The FGC spectrum is broader and peaks at the 25 th harmonic. In both gas cells, the harmonic intensity drops rapidly by 2 orders of magnitude between the 29th and 39th harmonics. In contrast, the spectrum of the PV peaks at the 19th harmonic, but has a broad plateau, with similar intensities in the range of the 15th-23rd harmonics. A rapid cutoff is observed past the 23rd harmonic. For all three sources, the harmonic spectrum exhibits a local minimum at the 37 th harmonic which can be attributed to the Cooper minimum $(\sim 50 \mathrm{eV})$ in argon [26,27]. This trend was observed to be independent of laser intensity.

We show that the differences in the harmonic spectra of the gas cells and the gas jet are a result of interplay between phase matching conditions and absorption of the harmonics. Efficient generation of high harmonics depends on three length parameters [13]; medium length $L_{\text {med }}$, absorption length $L_{\mathrm{abs}}=1 / \rho \sigma$, and coherence length $L_{\mathrm{coh}}=\pi / \Delta k$. The phase mismatch between the $q$ th harmonic and the fundamental fields is given by $\Delta k=k_{q}-q k_{0}$, where $k_{q}$ and $k_{0}$ are harmonic and fundamental wave vectors, respectively. In 


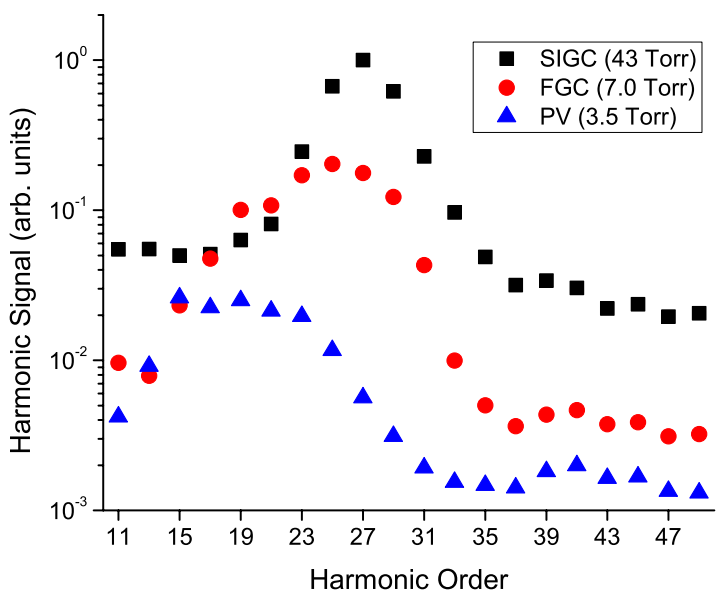

FIG. 5. (Color online) Observed high harmonic spectrum for different gas sources. The laser intensity is $3 \times 10^{14} \mathrm{~W} \mathrm{~cm}^{-2}$ for the semi-infinite gas cell (black squares) and the finite gas cell (red circles). The pulsed valve data (blue triangles) were recorded with a laser intensity of $6 \times 10^{14} \mathrm{~W} \mathrm{~cm}^{-2}$.

free space, the propagation constant of light with vacuum wavelength $\lambda$ in a neutral gas medium is given by

$$
k=\frac{2 \pi n_{g}(\lambda)}{\lambda},
$$

where the index of refraction is $n_{g}=1+P \delta(\lambda)$, with the pressure, $P$, in atmospheres and the gas dispersion function, $\delta(\lambda)$, is given by a suitable empirical relationship [28]. In general, empirical relationships are difficult to deduce beyond the UV, and we use calculated data [29] for wavelengths shorter than $140 \mathrm{~nm}$. For sufficiently large laser intensities partial ionization of the medium occurs and the index of refraction is modified as

$$
n_{g}=1+(1-\eta) P \delta(\lambda)+(1-\eta) n_{2} I-\eta P N_{\mathrm{atm}} r_{e} \lambda^{2} / 2 \pi,
$$

where $N_{\text {atm }}$ is the number density at atmospheric pressure, $\eta$ is the ionization fraction, and $r_{e}$ is the classical electron radius. The nonlinear index of refraction, $n_{2} \sim 9.9 \times 10^{-18} \mathrm{~cm}^{2} \mathrm{~W}^{-1} \mathrm{~atm}^{-1}$ [30], has negligible effect at our intensities. The phase mismatch then becomes

$$
\Delta k=\eta P N_{\mathrm{atm}} r_{e}\left(q \lambda_{0}-\lambda_{q}\right)-\frac{2 \pi(1-\eta) P}{\lambda_{q}}\left[\delta\left(\lambda_{0}\right)-\delta\left(\lambda_{q}\right)\right] .
$$

The ionization fraction, $\eta$, was calculated using the ADK theory of tunnel ionization [31] at similar intensities to those used in the experiment. For an argon atom and a pulse duration of $35 \mathrm{fs}, \eta$ is $\sim 0.08$ at $4 \times 10^{14} \mathrm{~W} \mathrm{~cm}^{-2}$ and $\sim 0.42$ at $7 \times 10^{14} \mathrm{~W} \mathrm{~cm}^{-2}$. These values were used in calculating $\Delta k$. We then calculated the number of $q$ th harmonic photons per unit time and area emitted on-axis, which is proportional to

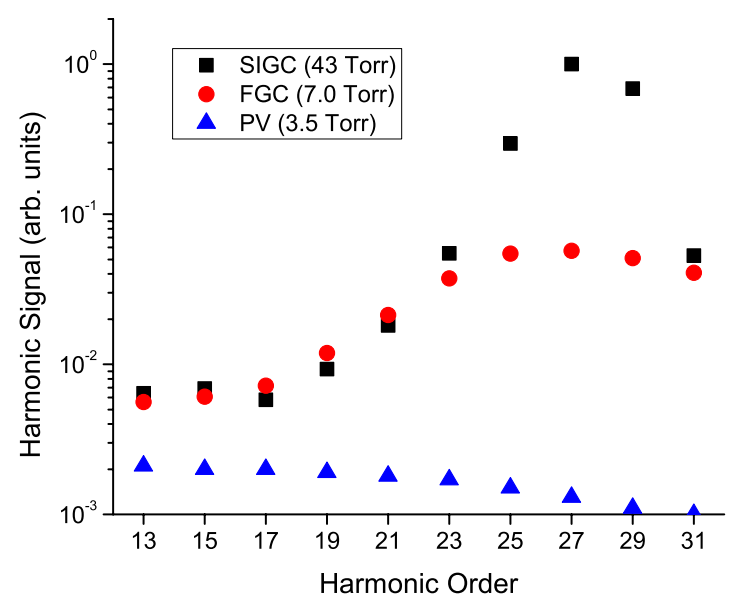

FIG. 6. (Color online) Theoretical harmonic signal for the SIGC (black squares), FGC (red circles), and PV (blue triangles) calculated using Eq. (5). The ionization fraction is $\eta=0.08$ for the SIGC and FGC, and $\eta=0.42$ for the PV, corresponding to laser intensities of $I=4 \times 10^{14} \mathrm{~W} \mathrm{~cm}^{-2}$ and $I=7 \times 10^{14} \mathrm{~W} \mathrm{~cm}^{-2}$, respectively.

$$
\begin{aligned}
N_{\text {out }} \propto & \rho^{2} A_{q}^{2} \frac{4 \rho^{2} L_{\text {abs }}^{2}}{1+4 \pi^{2}\left(L_{\text {abs }}^{2} / L_{\mathrm{coh}}^{2}\right)}\left[1+\exp \left(-\frac{L_{\text {med }}}{L_{\text {abs }}}\right)\right. \\
& \left.-2 \cos \left(\frac{\pi L_{\text {med }}}{L_{\text {coh }}}\right) \exp \left(-\frac{L_{\text {med }}}{2 L_{\text {abs }}}\right)\right]
\end{aligned}
$$

where $A_{q}$ is the amplitude of the atomic response approximated to be $(1-\eta) I^{3}$, where $I$ is the laser intensity.

The theoretical spectra for the gas cells and the gas jet are calculated with Eqs. (4) and (5) and plotted in Fig. 6, using similar intensities as in the experiment. We see a qualitative agreement between our experimental results and the theoretical calculation. Note that harmonics higher than the 31st are not included in the theoretical spectra due to the lack of dispersion data. In the case of the PV, the HHG efficiency is low due to lower number densities. The number density can be increased to some extent by increasing the gas load, but there are strict physical limitations on how much gas pressure the PV can accommodate. Since the medium length of $\sim 1 \mathrm{~mm}$ is much shorter than the typical absorption length, even in the strongly absorbing region near H15 $(\sim 3 \mathrm{~mm}$ at 3.5 Torr), absorption of the generated harmonics by the medium is negligible. As the ionization fraction increases with intensity, phase matching for higher harmonics cannot be achieved, resulting in the observed decrease in HHG efficiency.

The harmonic distribution for the two gas cells can be explained in terms of absorption and phase matching. The higher gas densities in the cells lead to lower absorption lengths, especially around the 15th harmonic. As a result, lower order harmonics are absorbed by the medium in which they are produced. Higher order harmonics have lower efficiency due to phase matching problems caused by ionization. As the ionization fraction increases, the peak in the spectral distribution shifts toward lower harmonics. The interplay between phase matching and absorption gives rise to the observed distribution with a peak around the 27th harmonic. 


\section{CONCLUSION}

By monitoring the photoionization of a background gas by the xuv photons produced via $\mathrm{HHG}$, we have made a direct comparison of high harmonic generation in an argonfilled pulsed valve, a $11 \mathrm{~mm}$ finite gas cell, and a semiinfinite gas cell. Under optimal pressure conditions, we have shown that the harmonic efficiency in finite and semi-infinite gas cells is, respectively, 50 and 100 times that which is produced in pulsed valve. The strongest harmonic emission was obtained with the semi-infinite gas cell, which had an estimated conversion efficiency of $\sim 10^{-4}$. We show that the spectral distributions in both the gas jet and the cells are different due to the role of phase matching and absorption. Our results reaffirm that large conversion efficiencies cannot be achieved by simply using higher atomic densities and longer medium lengths, because, while the efficiency depends quadratically on these two parameters, it also depends on the negative exponential of their product. As a result, absorption of harmonics starts to play a critical role along with phase matching. Further enhancements in HHG efficiency depend on absorption management. By far the most promising technique to efficiently produce high harmonics employs successive sources to modulate the atomic number densities along the interaction length in order to achieve quasiphase matching while simultaneously minimizing absorption [32]. This and similar density modulation techniques might enable one to increase the harmonic flux and thereby realize experiments in xuv spectroscopy and attosecond domains.

\section{ACKNOWLEDGMENTS}

Funding for the present work was provided by the Natural Science and Engineering Research Council. The authors thank Daniel Comtois of l'Institut National de la Recherche Scientifique (INRS) for assistance during experiments conducted at Advanced Laser Light Source. The authors would also like to thank David Villeneuve and Paul Corkum at the National Research Council Canada for fruitful discussions and for allowing us to conduct preliminary experiments at their facility. J.-P.B. would like to thank Jessica Brichta and Renée Gibbins for assistance in the preparation of this paper.
[1] A. McPherson, G. Gibson, H. Jara, U. Johann, T. S. Luk, I. McIntyre, K. Boyer, and C. K. Rhodes, J. Opt. Soc. Am. B 4, 595 (1987)

[2] J. J. Macklin, J. D. Kmetec, and C. L. Gordon III, Phys. Rev. Lett. 70, 766 (1993).

[3] P. B. Corkum, Phys. Rev. Lett. 71, 1994 (1993).

[4] M. Lewenstein, Ph. Balcou, M. Y. Ivanov, A. L'Huillier, and P. B. Corkum, Phys. Rev. A 49, 2117 (1994).

[5] N. A. Papadogiannis, L. A. A. Nikolopoulos, D. Charalambidis, G. D. Tsakiris, P. Tzallas, and K. Witte, Phys. Rev. Lett. 90, 133902 (2003).

[6] M. Hentschel, R. Keinberger, Ch. Spielmann, G. A. Reider, N. Milosevic, T. Brabec, P. B. Corkum, U. Heinzmann, M. Drescher, and F. Krausz, Nature (London) 414, 509 (2001).

[7] J. Itatani, J. Levesque, D. Zeidler, H. Niikura, H. Pepin, J. C. Kieffer, P. B. Corkum, and D. M. Villeneuve, Nature (London) 432, 867 (2004).

[8] R. Ganeev, M. Suzuki, M. Baba, H. Kuroka, and T. Ozaki, Opt. Lett. 30, 768 (2005).

[9] E. Takahashi, Y. Nabekawa, T. Otsuka, M. Obara, and K. Midorikawa, Phys. Rev. A 66, 021802(R) (2002).

[10] J.-F. Hergott, M. Kovačev, H. Merdji, C. Hubert, Y. Mairesse, E. Jean, P. Breger, P. Agostini, B. Carré, and P. Salières, Phys. Rev. A 66, 021801(R) (2002).

[11] E. Takahashi, Y. Nabekawa, and K. Midorikawa, Appl. Phys. Lett. 84, 4 (2004).

[12] P. Salières, A. L'Huillier, and M. Lewenstein, Phys. Rev. Lett. 74, 3776 (1995).

[13] E. Constant, D. Garzella, P. Breger, E. Mével, Ch. Dorrer, C. LeBlanc, F. Salin, and P. Agostini, Phys. Rev. Lett. 82, 1668 (1999).

[14] Y. Tamaki, J. Itatani, Y. Nagata, M. Obara, and K. Midorikawa, Phys. Rev. Lett. 82, 1422 (1999).

[15] J. R. Sutherland, E. L. Christensen, N. D. Powers, S. E. Rhynard, J. C. Painter, and J. Peatross, Opt. Express 12, 4430 (2004).
[16] E. A. Gibson et al., Science 302, 95 (2003).

[17] R. Bartels, S. Backus, E. Zeek, L. Misoguti, G. Vdovin, I. P. Christov, M. M. Murnane, and H. C. Kapteyn, Nature (London) 406, 164 (2000).

[18] T. Pfeifer, R. Kemmer, R. Spitzenpfeil, D. Walter, C. Winterfeldt, G. Gerber, and Ch. Spielmann, Opt. Lett. 30, 1497 (2005).

[19] I. J. Kim, C. M. Kim, H. T. Kim, G. H. Lee, Y. S. Lee, J. Y. Park, D. J. Cho, and C. H. Nam, Phys. Rev. Lett. 94, 243901 (2005).

[20] H. W. Liepmann and A. Roshko, Elements of Gasdynamics (Wiley, New York, 1957).

[21] D. H. Levy, Annu. Rev. Phys. Chem. 31, 197 (1980).

[22] L. A. Lompré, M. Ferray, A. L'Huillier, X. F. Li, and G. Mainfray, J. Appl. Phys. 63, 1791 (1988).

[23] S. M. Hankin, D. M. Villeneuve, P. B. Corkum, and D. M. Rayner, Phys. Rev. Lett. 84, 5082 (2000).

[24] M. Nisoli, G. Sansone, S. Stagira, C. Vozzi, S. De Silvestri, and O. Svelto, Appl. Phys. B: Lasers Opt. 75, 601 (2002).

[25] J. W. Gallagher, C. E. Brion, J. A. R. Samson, and P. W. Langhoff, J. Phys. Chem. Ref. Data 17, 9 (1988).

[26] J. W. Cooper, Phys. Rev. 128, 681 (1962).

[27] B. K. McFarland, J. P. Farrell, M. Guhr, and P. H. Bucksbaum, Lasers and Electro-Optics, 2008 and 2008 Conference on Quantum Electronics and Laser Science (CLEO/QELS 2008), 2008 (unpublished), pp. 1-2.

[28] M. J. Weber, Handbook of Optical Materials (CRC, Cleveland, 2002).

[29] G. Liggett and J. S. Levinger, J. Opt. Soc. Am. 58, 109 (1968).

[30] H. J. Lehmeier, W. Leupacher, and A. Penzkofer, Opt. Commun. 56, 67 (1985).

[31] M. V. Ammosov, N. B. Delone, and V. P. Krainov, Sov. Phys. JETP 64, 1191 (1986).

[32] J. Seres, V. S. Yakovlev, E. Seres, Ch. Streli, P. Wobrauschek, Ch. Spielmann, and F. Krausz, Nat. Phys. 3, 878 (2007). 